\title{
Etats-Unis : le débat sur la loi No Child Left Behind à l'occasion des élections présidentielles.
}

\section{Marion Latour}

\section{(2) OpenEdition}

1 Journals

Édition électronique

URL : https://journals.openedition.org/ries/1487

DOI : $10.4000 /$ ries. 1487

ISSN : 2261-4265

Éditeur

France Education international

\section{Édition imprimée}

Date de publication : 1 décembre 2004

Pagination : $12-14$

ISBN : 978-2-85420-564-0

ISSN : $1254-4590$

\section{Référence électronique}

Marion Latour, «Etats-Unis : le débat sur la loi No Child Left Behind à l'occasion des élections présidentielles. ", Revue internationale d'éducation de Sèvres [En ligne], 37 | décembre 2004, mis en ligne le 18 novembre 2011, consulté le 08 juillet 2021. URL : http://journals.openedition.org/ries/1487 ; DOI : https://doi.org/10.4000/ries.1487

Ce document a été généré automatiquement le 8 juillet 2021.

(c) Tous droits réservés 


\title{
Etats-Unis : le débat sur la loi No Child Left Behind à l'occasion des élections présidentielles.
}

\author{
Marion Latour
}

1 Les dernières élections américaines ont mis en lumière les engagements des deux principaux candidats dans le domaine éducatif. Même si l'éducation n'a pas été au centre du débat électoral, Georges W. Bush, le président républicain sortant, et John Kerry, son rival démocrate, ont pris position sur la grande réforme mise en place par l'administration Bush, la loi No Child Left Behind ACt, NCLB'. Rappelons que la loi a été votée par les républicains comme par les démocrates. Aujourd'hui, plus de deux ans et demi après sa promulgation, continue-t-elle de rallier les deux partis?

2 Loi fédérale ${ }^{2}$, elle s'applique uniquement aux établissements publics qui reçoivent des fonds fédéraux de lutte contre la pauvreté, ce qui concerne dans les faits près de $70 \%$ des établissements du pays. Elle repose sur quatre piliers ${ }^{3}$ :

- une obligation de progrès, mesurés par des tests annuels pour les enfants entre huit et treize ans en mathématiques et en lecture ;

- une liberté accrue pour les États et les districts dans l'utilisation des fonds fédéraux en fonction de leurs besoins spécifiques et dans les moyens d'atteindre les objectifs,

- un choix d'établissement plus grand laissé aux parents,

- des méthodes d'enseignement éprouvées.

Chaque année, les établissements doivent attester de résultats en progrès, au niveau de l'établissement lui-même et à l'échelle du district et de l'État et cette progression doit être analysée en fonction des groupes sociaux et ethniques. Les États ont la responsabilité de publier les résultats des établissements, des districts et de l'État et d'expliquer comment, concrètement, ils s'emploient à atteindre les objectifs assignés. Les établissements en difficulté reçoivent des fonds fédéraux pour proposer des mesures d'accompagnement, telles que le soutien scolaire et les cours du soir. Au-delà de deux années consécutives sans amélioration, les parents peuvent changer leurs enfants d'établissement à l'intérieur du district, les frais de déplacement étant pris en 
charge par celui-ci. Au terme de cinq années, des actions correctives sont prises, qui peuvent aller jusqu'à un changement structurel de l'établissement. Le ministère fédéral accompagne la réforme sur le terrain (publication de guides, ressources sur le web...) et finance des programmes pédagogiques pour rendre plus efficace l'enseignement et l'apprentissage, en particulier de la lecture ${ }^{4}$, préoccupation affichée de Georges W. Bush. Comment cette loi a-t-elle été appliquée depuis janvier 2002 ?

4 Au début de l'administration de G. W. Bush, seuls onze États avaient mis en place une politique de responsabilisation appelée accountability ${ }^{5}$ alors que la réglementation de 1994 l'imposait déjà. Dix-huit mois après l'approbation du NCLB, les cinquante États plus le district de Columbia et Puerto Rico se sont dotés de programme d'accountability. Pressée par un certain nombre d'États qui refusaient de se soumettre à la réglementation fédérale ${ }^{6}$, l'administration Bush a accepté à plusieurs reprises d'assouplir les règles de sa réforme, notamment concernant le nombre d'enseignants qualifiés et la non prise en compte des élèves maîtrisant insuffisamment l'anglais ; cette dernière concession concerne 5,5 millions d'élèves, soit $12 \%$ des effectifs. En 2004, les performances en lecture et mathématiques ont montré que $25 \%$ des établissements avaient un niveau inférieur aux exigences requises, contre $28 \%$ en 2003. S'ils témoignent d'un progrès, ces chiffres ont suscité la polémique car certains estiment que ces résultats sont justement le fruit de l'assouplissement des règles, les établissements pouvant exempter les élèves qui pèsent sur les résultats. Par ailleurs, ils estiment que l'on a surtout appris aux élèves à bien passer les tests. Le ministre fédéral de l'Éducation défend l'efficacité du NCLB, chiffres à l'appui : alors que les résultats en lecture pour la $4^{\mathrm{e}}$ classe, équivalent du CM1 en France, avaient stagné entre 1992 et 2000 , ils ont gagné cinq points ces trois dernières années; les progrès sont sensibles en mathématiques et en lecture chez les élèves noirs américains et ceux d'origine hispanique .

5 Le changement d'établissements est plus utilisé que par le passé, même si la demande dépasse de manière significative l'offre ${ }^{8} .70000$ élèves ont pu changer d'établissements en 2004 , soit 2 à $3 \%$ des élèves. Ce système a une limite: dans les districts où un pourcentage élevé d'établissements a échoué aux tests, il est techniquement inapplicable de permettre aux élèves de s'inscrire dans des établissements d'un meilleur niveau. C'est le cas dans le district de Washington, où $46 \%$ des établissements n'ont pas fait preuve de progrès suffisants. Seul le transfert dans des écoles de banlieue riches pourrait être efficace, mais cette solution est inenvisageable politiquement. Comme le souligne la revue Education Next dans son numéro d'automne 2004, on se heurte désormais à une ségrégation géographique.

6 En cas de deuxième mandat, G.W. Bush entend poursuivre la réforme du NCLB en étendant les tests annuels jusqu'à la onzième classe, l'équivalent de la première dans le système éducatif français. Il souhaite concentrer l'effort éducatif sur le secondaire, en revalorisant le diplôme qui clôt la fin des études secondaires et en préparant mieux les élèves à la vie professionnelle et aux études supérieures. John Kerry, lui, veut également favoriser le secondaire, avec l'objectif qu'un million d'élèves supplémentaires entrent à l'université.

7 Si John Kerry est attaché aux standards et au principe de responsabilisation, il se montre très critique à l'égard du NCLB. Il s'oppose au caractère automatique des sanctions quand une école ne remplit pas l'ensemble des critères et reproche à G.W. Bush de n'avoir pas révisé le budget du NCLB de 18 à 22 milliards de dollars comme 
promis. Il s'engage à annuler les privilèges fiscaux accordés par son rival aux plus riches, afin de constituer un fonds pour l'éducation qui récolterait 200 milliards de dollars en dix ans.

G. W. Bush met en avant l'augmentation du budget fédéral destiné aux établissements : sur l'année scolaire 2003-04, le gouvernement fédéral représente $8,2 \%$ du total des dépenses en éducation pour les niveaux primaire et secondaire, contre $5,7 \%$ en 1990-91, soit une progression de plus d'un tiers9. Si le budget 2005 est accepté, les dépenses en éducation auront augmenté de 35,8 \% depuis 2001.

Le NCLB s'inscrit dans la continuité des politiques de responsabilisation que poursuit le gouvernement depuis 1983, date à laquelle fut publié le rapport A nation at risk. Les États-Unis se heurtent aux mêmes difficultés que d'autres pays occidentaux : diversité des publics scolaires et niveau insuffisant dans des matières fondamentales (lecture, mathématiques). La loi que le gouvernement de G.W. Bush a élaborée durant son premier mandat est une réponse jugée insuffisante dans ses moyens par les démocrates ; les Républicains donnent pour échéance l'année 2014 pour atteindre les objectifs qu'ils se sont fixés.

\section{NOTES}

1. Littéralement : aucun enfant laissé de côté. Voir le $n^{\circ} 30$ de la Revue internationale d'éducation.

2. Aux États-Unis, l'éducation est en priorité du ressort des États et des districts. Cette loi a donc une dimension politique très forte.

3. Consulter le site officiel de la loi : http://www.ed.gov/nclb/

4. Le ministère fédéral de l'Éducation a formé plus de 75000 enseignants à une nouvelle pratique pédagogique plus scientifique, qui bénéficiera à 1,4 million d'élèves répartis dans près de 4300 établissements du pays.

5. Par accountability, on entend que la réussite des élèves est de la responsabilité des établissements. La responsabilisation se décline en quatre mesures: les critères (standards), définissant les compétences que les élèves doivent acquérir ; les tests, pour mesurer la maîtrise des standards; la publication des résultats et d'un certain nombre d'indicateurs pour chaque établissement; l'incitation, qui peut prendre des formes diverses, de sanctions graduées à des récompenses pour les établissements performants. D'après Denis Meuret, "Les réformes de l'éducation aux États-Unis ", septembre 2004, sur le site de l'Institut de recherche sur l'éducation de l'université de Bourgogne (IREDU) : http://www.u-bourgogne.fr/IREDU/2004/04088.pdf

6. C'est le cas en particulier d'Hawaii, de la Virginie, de l'Utah, du New Hampshire, du Maine, du Vermont, de l'Arizona, du Nebraska et du Wisconsin.

7. Voir la rubrique affichant les résultats par États: http://www.ed.gov/nclb/overview/ importance/difference/index.html

8. Rapport publié en octobre 2003 consultable sur : http://www.brookings.edu/dybdocroot/gs/ brown/bc_report/2003/2003report.pdf

9. Source : Document consultable sur le site du ministère fédéral de l'éducation, intitulé « 10 facts about K-12 education funding » : http://www.ed.gov/about/overview/fed/10facts/10facts.pdf 
INDEX

Mots-clés : réforme de l'enseignement, système éducatif

Index géographique : États-Unis

\section{AUTEUR}

MARION LATOUR

Documentaliste au centre de ressources du CIEP 